PHYSICAL REVIEW D 93, 089907(E) (2016)

\title{
Erratum: Quantum field theoretical description for the reflectivity of graphene [Phys. Rev. D 91, 045037 (2015)]
}

\section{Bordag, G. L. Klimchitskaya, V. M. Mostepanenko, and V. M. Petrov}

(Received 12 April 2016; published 20 April 2016)

DOI: $10.1103 /$ PhysRevD.93.089907

There are errors in several mathematical expressions of this paper which have no influence on the obtained results, but should be corrected for future applications of the developed formalism.

Thus, on p. 4 (right column) in the third line above the page bottom, one should replace $\omega=-i \xi$ with $\omega=i \xi$.

In Eq. (36) the factor $i \operatorname{sign}\left[\omega-\lambda \Gamma\left(q_{\perp}\right)\right]$ should be replaced with $-i$.

As a result, in the second line on the right-hand side of Eq. (43) the factor $i$ should be replaced with $-i$.

The above sign corrections lead to the following corrections in Secs. IV and V.

The sign minus should be added in front of the right-hand side of Eqs. (68)-(70), (81), and (82).

Equation (83) should be replaced with

$$
\operatorname{Im} \Delta_{T} \Pi_{00}\left(\omega, k_{\perp}\right) \approx-\pi \alpha \frac{k_{\perp}^{2}}{\tilde{p}\left(\omega, k_{\perp}\right)} \frac{2}{e^{\frac{\omega}{2 T}}+1} .
$$

In Eqs. (89) and (95) the factor 2 in front of the imaginary parts should be replaced with $\omega /(4 T)$.

The factor $\pi \alpha$ in Eq. (90) and the term $\pi \alpha$ in Eq. (96) in both numerators and denominators should be multiplied by $\omega /(8 T)$.

In Eqs. (91), (92), and (97) the quantity $\pi^{2} \alpha^{2}$ in numerators should be multiplied by $\omega^{2} /\left(64 T^{2}\right)$ and the quantity $\pi \alpha$ in denominators by $\omega /(8 T)$.

The first line of Eq. (93) should be replaced with

$$
\operatorname{Im} \Delta_{T} \Pi_{\mathrm{tr}}\left(\omega, k_{\perp}\right) \approx \pi \alpha \frac{p^{2}\left(\omega, k_{\perp}\right)+\tilde{p}^{2}\left(\omega, k_{\perp}\right)}{\tilde{p}\left(\omega, k_{\perp}\right)} \frac{2}{e^{\frac{\omega}{2 T}}+1} .
$$

In the second line of Eq. (94) the factor -2 in front of $i$ should be replaced with $\omega /(4 T)$.

All the above changes apply to small correction terms and have no impact on the respective figures.

There are also typos in Eq. (77) and in the first line of Eq. (78). In Eq. (77) the factor $T^{3} / \omega^{4}$ should be replaced with $T^{3} / \omega^{2}$. In the first line of Eq. (78) one should replace $T^{3} / \omega^{3}$ with $T^{3} / \omega^{2}$ in both the numerator and denominator. 